\title{
Demoscene Platforms: A Case Study on the Adoption of Home Computers
}

\author{
Markku Reunanen $^{1}$ and Antti Silvast ${ }^{2}$ \\ ${ }^{1}$ University of Art and Design Helsinki \\ markku.reunanendiki.fi \\ ${ }^{2}$ University of Helsinki \\ antti.silvast@iki.fi
}

\begin{abstract}
This paper discusses the adoption of new technology by a hobbyist community known as the demoscene. The demoscene is chiefly a European community that originates from the mid-1980s and continues to exist even today. During its twenty years of existence, the demoscene has had to react to several changes in the field of information technology, in particular new hardware and software platforms. Based on the contemporary communication found in disk magazines and Internet forums we present case examples of the transitions and analyze the adoption processes. At large, the observations made serve as examples of the rich and unexpected ways in which the home computers were domesticated since the early 1980s.
\end{abstract}

Keywords: Demoscene, Computer hobbyists, Diffusion of innovations, Multimedia.

\section{Introduction}

The demoscene is a community that creates digital art with home computers. It has its roots in the late 1970s home computer revolution and software piracy. The demoscene-or just the scene-has traditionally been a male-dominant hobby, popular in Europe and especially in the Nordic countries. The aim of this study is to provide the reader an overview of the community and its relationship with the ever-changing world of computing. Ultimately, the demoscene and its practices serve as examples of the rich and unexpected ways of living with computers, never anticipated by the original manufacturers.

Any new technology needs to fit into already existing relationships and practices. In the context of the demoscene, a typical example of this would be a new computer like a Commodore 64 or Amiga or PC appearing in the market. By studying how the demosceners react to new platforms, we aim to show that new computers do not get judged only by technical features or market price. Rather, the reaction to new computers has to do with inclusion: it is important for the sceners to estimate which material objects and persons can belong to the scene and which cannot.

The demoscenes' communication has not been studied from this perspective before. On a different level, our study aims to describe a self-reflective community of technology users. We claim that communities of computer users are actively debating about those social relationships, practices, and technologies that account for the community itself. 


\section{Starting Points for the Study}

To understand the demoscene and its development it is necessary to place it in a historical context. The contemporary technology and its possibilities are fundamental for such a culture. However, they alone do not explain why an international community of demo groups would emerge in the late 1980s. In the following, we present the reader a historical overview of the era and position our work in the context of demo research conducted by other researchers.

\subsection{Historical Frame}

The home computer revolution of the late 1970s and the early 1980s was a necessary precondition for the birth of the demoscene. Affordable computers appeared in stores and for the first time in history were available to the masses. This commercial and technological development was not enough in itself - there had to be a need and interest to buy one. The early attitudes towards computers were often controversial: advertisements and governmental plans typically emphasized educational values, whereas in reality gaming was the most popular use from the very beginning [18]. The loose attitudes towards copyrights lead to an extensive software piracy, which in turn lead to the birth of the pirate scene with its crack intros, later evolving to computer demos $[14,17]$. Cracking in this context refers to the removal of copy protections of commercial software.

The first popular home computers such as Sinclair Spectrum and Commodore VIC-20 did not yet feature extensive sound or graphics capabilities. The low amount of memory was also a limiting factor. The first demoscene computer was Commodore 64, introduced in 1982, featuring advanced graphics and sound for its time. Its popularity was not shadowed until the late 1980s. For example, in Finland C64 was advertised as "Tasavallan tietokone"-_The Computer of the Republic" [18]. The other 8-bit computers of the era such as MSX and Amstrad CPC sold well in certain countries but did not attract the early demosceners in great numbers, presumably due to their lesser popularity and a weaker pirate scene.

The following important model of Commodore computers was Amiga 1000 introduced in 1985, followed by Amiga 500 (1987), which was a more compact and affordable model suitable for home users [1]. From the home computer perspective, the major competitor was Atari ST, released in 1985, which was able to sustain a modest demoscene of its own. Commodore retained its position amongst the hobbyists with its Amiga line of computers until the early nineties. By that time, the border between home and business computers had mostly faded away. IBM PC compatibles started appearing at homes and after the Commodore bankruptcy in 1994 [1] there was little commercial competition left. We examine the effects of these changes on the demo hobbyists in further detail in Section 4.

\subsection{Related Work and Criticism}

As we have overviewed in our online demoscene research bibliography [15], media researchers, sociologists, and cultural historians have already shown some interest in the demoscene. For the purpose of this article, we separate these studies to two domains. The first way of researching the scene has been to view it as artistic activity, 
which makes it the subject of art research. "Demoscene culture", in this use, is likened to other artforms, or it is even elevated to an artform of its own. This has meant looking at the demoscene as "art of the real-time" [20, 14], as a form of musical hobby [12] or as an emerging digital art form [19]. There is also a second way to measure the culture in the demoscene: as a particular way of life. This perspective relates to sociology, cultural studies, and cultural history. The researchers have discussed demoscene as youth culture or counter culture [17], multimedia hacker culture [6] or as a gendered community [9].

We admit that these studies have opened up demoscene for discussions in the mentioned research domains. In doing this, they have however taken a very abstract and often an outsider perspective to the scene. Another major pitfall of these studies has been the tendency to write "the history of the winners", often based on anecdotal evidence. Narrowing the research to famous groups and competition winners leads to a biased view of the phenomenon, since the majority of members and artifacts of the scene fall outside this winners' category. Overall, we feel that the real live action of being in the scene has been neglected thus far. In this article, we stress that the demoscene is always something active; as community, it is a collection of social relationships, practices and technologies [10], whose composition must be discussed by the members in order to keep it afloat.

\section{Material and Methods}

There is a wide variety of artifacts produced in the context of the demoscene. Examples of these are demos, intros (small demos), disk magazines, text files, pictures and tunes, which have already been studied $[17,20]$. Additionally the communication between the scene members produces messages in modem-based bulletin board systems (BBS), newsgroups, disk magazines and message boards. The demoscene artifacts too convey meanings, but to understand the cultural values and reasons for phenomena it is reasonable to choose communication, because in it the active construction of common meanings is made more explicit. Various Internet archives such as demo sites proved to be a valuable source for the desired material-gathering the same information fifteen years ago would have required a great deal more time and an extensive network of contacts in the community.

The skipping of material on Atari ST, MSX, Sinclair Spectrum and other small scenes was a conscious choice. Firstly, the three most active platforms (Commodore 64, Amiga, and PC) and their changes are easier to study due to the good availability of source material. The material also represents a wider population. Secondly, since our focus is on transitions and reactions instead of the particular properties of the different scenes the actual selection of platforms is fairly insignificant.

\subsection{Disk Magazines}

Disk magazines — or diskmags for short—are interactive electronic magazines, which were originally distributed on diskettes in copy parties. The structure of a diskmag roughly mimics the structure of an ordinary magazine or a newspaper: there is an editorial, news, rumors, advertisements, interviews, and articles on topics that interest the readers. Charts are an important part of diskmags. In the charts, the most popular 
groups, coders, swappers, musicians, graphics artists ("graphicans") and demos are ranked either by voting or according to the editor's personal preferences.

Several properties of diskmags make them a valuable source for researchers. Sceners write them to sceners, meaning the topics reflect the interests of the community. For the same reason the opinions stated are typically not tuned down to please the outsiders. The articles are also contemporary, providing a peek to the phenomena of the time not colored by nostalgia, which would be the case if we interviewed the writers today. The rumors and speculation in diskmags are especially useful for tracing contemporary understanding and debates. Those seeking for historical facts should however note that the mags' factual content is not very reliable, because it is characterized by differing interpretations and competition of groups and cliques.

For this study we chose four diskmags as the main sources of information. The large amount of diskmags made the selection a difficult one but based on the opinions of hobbyist sources, we picked Sex'n'Crime (Commodore 64), Zine (Amiga), R.A.W. (Amiga), and Imphobia (PC). The 45 issues analyzed cover the period from 1989 to 1996. In the 1980 s there were earlier disk mags as well but the period covered by Sex'n'Crime (1989-1990) is of particular interest because of the Commodore 64Amiga migration. At the other end of the span, mid-nineties, the diskmags started to lose their status as a communication channel because of the Internet.

\subsection{Supporting Material}

Since the mid-1990s, the scene has increasingly started to use the Internet for the exchange of thoughts. Already in 1993, there was an article about the net in the Imphobia diskmag. To support and contrast the observations made from the diskmags, we used the ample archives of Usenet newsgroups alt.sys.amiga.demos and comp.sys.ibm.pc.demos as supporting material. Especially the latter newsgroup was highly active up until 2002: according to Google Groups, there were over 10,000 messages yearly during the most active period [2]. Reading and analyzing all the messages would have been a colossal task so we chose to limit the scope to relevant topics dealing with the adoption of new technologies.

Two additional resources used were competition results from the four biggest yearly parties (scener meetings) and pouet.net, a popular demoscene website. In contrast to the qualitative data of diskmags and newsgroups, the data obtained from competition results was quantitative by nature. The parties chosen were Assembly (Finland), The Party (Denmark), The Gathering (Norway) and Mekka \& Symposium (Germany), during the period 1992-2002. Before 1992 the parties were smaller in scale and organized more often, according to Polgar [14] and the reports found in the Sex'n'Crime diskmag. The sample obtained from the party results consisted of 2094 productions.

\subsection{Methods}

Our observation of the text articles and discussions was based on distinctions and associations. We observed the construction of oppositions: what kinds of practices belong to the scene and which outside the scene? What associates with "elite" sceners (i.e. valuable), what with "lame" uses and users of computers (i.e. not valuable)? What marks the boundaries of the scene use and other use of computers? 
When dealing with the quantitative data of the party results and productions the goal was to facilitate visual interpretation. Five variables were chosen: amount of Commodore 64 demos, Amiga demos, Amiga intros, PC demos and PC intros each year. We obtained the totals by summing the quantities from each party. Unfortunately, the limitations of the data set reduce the accuracy of the result. Firstly, the competitions in some Assembly parties were limited to only 15 entries because of a preselection. Secondly, Mekka \& Symposium party appeared only in 1996 so its contribution is not present in the earlier totals. The latter weakness is somewhat countered by the effect of Mekka \& Symposium growing from a small party at the expense of The Gathering and The Party.

\section{The Major Transitions}

The notion of a major transition here refers to a significant development in the information technology industry and the reactions of the demoscene to it. This section is divided according to the dominant platform of the era. It should be noted that this division is merely a simplification of the actual events: the different eras are overlapping and greatly more complex and detailed than what the threefold model would suggest.

In his book on the diffusion of innovations Rogers presents five distinctive groups of adopters: innovators, early adopters, early majority, late majority, and laggards [16]. Our observations of the transitions of the demoscene revealed a similar pattern: innovators try out a new platform early on but the demos they make are mere curiosities at that time. Influential persons and groups-early adopters-migrating to the new platform eventually start dragging the early majority with them. The late majority adapts because of practical reasons and only the laggards remain opposed to the transition. The retro movement has its point of reference in the past. In the demoscene circles, the phenomenon is now called "oldschool" or "oldskool" (also used by Tasajärvi et al. [20] to refer to the first era of the scene).

\subsection{Commodore 64}

Our most important source, Sex'n'Crime, was a Commodore 64 diskmag published by the group Amok during 1989-1990. The first impression the reader gets from Sex'n'Crime is that the Commodore 64 cracker/demo scene of the late 1980s was a hostile environment. Numerous accusations, rumors, and news about wars between groups appear practically in every issue of the diskmag. The rhetorical style found in the articles is emotionally loaded (both positive and negative) and occasionally downright harsh. As an example of the style, in Sex'n'Crime \#21 (1990) OMG/Amok responded to a letter to the editor like this:

"Dear Roy of Dynamics, let me say this from the bottom of my heart: you are lame!"

The ranking of individuals and groups in the charts was a controversial subject, sparking heated discussion in the following issues. In some letters and interviews, you can sense dissatisfaction with the ongoing wars and unfriendliness but overall these opinions did not constitute a significant part of the discourse. The distinction 
between the legal and the illegal activities was vague: cracked games, police raids, pirate software swapping and legal demos still went hand in hand in 1990. Gruetzmacher provides a slightly different interpretation [4], claiming that the illegal and legal scene activities slowly drifted apart in the late 1980s.

The emergence of the Amiga line of computers in the mid-eighties naturally received attention from the Commodore 64 sceners. This was the first technological transition the young scene had to face. Surprisingly-in comparison to other changes documented in the following subsections-the reactions expressed in Sex'n'Crime were chiefly positive or neutral. Polgar, however, reports that there was opposition as well [14]. In the news section of Sex'n'Crime the gradual transition becomes apparent during 1989-1990 (news of people "going" to Amiga) but it was not portrayed negatively, while the style of the diskmag otherwise was controversial. An important factor was that while new, the Amiga was still a product of the same company as the familiar Commodore 64.

\subsection{Commodore Amiga}

Amiga 500 was the second major hardware platform of the demoscene, following the success of the Commodore 64. Similar to Commodore 64, Amiga started as a uniform platform where the software would run the same on all setups. However, later the newer models such as the Amiga 1200, released in 1992 with its new AGA (Advanced Graphics Architecture) chipset and faster processor fragmented the platform. Starting from 1992 both the R.A.W. diskmag and the alt.sys.amiga.demos newsgroup contain a multitude of messages about the incompatibility problems related to the new chipset - a new challenge that was there to stay. Another source of problems was the difference between the PAL and NTSC Amigas, which hindered the exchange of demos between Europe and the United States.

There are notable differences between the two diskmags analyzed: Zine, published from 1989 to 1991 was still extensively connected to the cracker tradition, whereas R.A.W. (1991-1996) was chiefly demoscene-oriented and more refined in its appearance. The historical connection between the two communities remained at least through swapper activities. As an example of this interplay, the contact section of R.A.W. \#5 (1993) still featured numerous advertisements for illegal swapping.

The first major transition faced by the Amiga sceners was the AGA in 1992 and the following two years. Incompatibility of software was only one facet of the transition. The opinions found in R.A.W. and the newsgroup varied from excitement to extreme resistance. The positive arguments were based on the new possibilities offered by the new hardware, whereas the opposition claimed that there was no challenge in doing demos on such powerful computers. Another argument used was the high price of the new machines, which placed them out of reach of many users and even lead to a supposed split of the scene. Rufferto/Covert Action Team summed up the views of the opposition in R.A.W. \#6 (1993):

“OK, you'll be able to do much better and faster routines, but everybody knows, that you're not one of the best coders then, you just have got one of the best Amigas!" 
In hindsight the real threat to the Amiga was neither the AGA nor the accelerator boards but the IBM PC. First in 1993 and increasingly thereafter the community members expressed concern of the future of their platform-earlier the status of the Amiga scene had been so strong that people would look down upon any rivals. In the heated newsgroup posts and diskmag articles there were numerous arguments for and against PCs. Interestingly even in the Amiga forums you could find favourable mentions of the PC, while the consensus was against the transition. A platform war kept raging in diskmags and newsgroups (including comp.sys.ibm.pc.demos). Some of the most common, often contradictory, arguments for and against the new platform were:

○ Amiga demos are better designed and programmed.

o PC hardware is not uniform.

o Windows/MS-DOS sucks, Amiga has a better operating system.

○ PC owners are followers of big companies.

○ Amiga has coprocessors for sound and graphics-either a positive or negative feature.

○ PC has more computing power / PC has too much computing power.

○ PC/Amiga owners just play games - used by both sides.

O PC is more suitable for texture mapping.

O PC has better graphics modes because of SVGA (Super VGA display cards).

○ Developers are leaving the Amiga, PC has more software.

Polgar [14] and Saarikoski [17] provided further discussion of the Amiga-PC clash. In addition, Saarikoski's remarks on the Finnish "machine wars" of the 1980s provide some means for understanding the juxtaposition [18].

\subsection{IBM PC}

The demoscene that formed on the IBM PC and compatible machines since the early 1990s cannot be treated as one single scene only. During its seventeen years, the PC demoscene has faced changes that can be compared to complete platform transitions.

Imphobia was an influential diskmag published in 1992-1996. The development of the magazine closely resembles the course of Zine and R.A.W. on Amiga: the early crude magazine evolved into a well-edited and impressive publication. In the early issues until 1993 references to software piracy could be found but after that the focus of the diskmag soon changed to the legal demoscene. The underdog status of the PC scene in comparison to Amiga was generally recognized and dealt with in varying manners: either by acknowledging the situation or by coming up with emotional or rational counterarguments. An interesting finding is that since Commodore 64 was not considered a threat any more, the writers considered it either outdated or- increasingly by time - held the coders in high regard for pushing the old computer beyond its limits.

A controversial theme occurring frequently in our source material was the increasing computational capacity of the PC. While some regarded it as an opportunity to make better demos there were opposing voices too, complaining that the 486 or Pentium did not require any skills from the programmer. These discussions started in Imphobia and comp.sys.ibm.pc.demos in 1993 and continued until at least 1995. Another, fiercer hardware-related disagreement concerning sound card support took 
place in 1994-1996. This time the controversy was about demos that only supported Gravis Ultrasound, which had gained a strong following among the PC sceners.

A new kind of transition started in 1995 (first mentioned in the newsgroup already in 1994) with the introduction of Windows 95. Until then Windows had been running on top of MS-DOS, the predominant operating system of the PC scene. This shift was technically different to the previous ones since it was about software, not hardware. The future of MS-DOS looked uncertain, which called for a reaction from the community. The response was highly emotional and at first mostly negative. We must specifically mention one of the many arguments used in the discussions: the emergence of Windows 95 would mean an end to direct hardware access, which had been a principal technique used in demos from the earliest days in order to achieve the best possible performance. Losing this control over the machine did not suit the existing practices. During 1995-1998, the status quo did not yet change, with most demos still released for the accepted MS-DOS platform. Several options such as OS/2, Linux and even an own operating system "DemOS" were considered but eventually the PC scene followed the industry and mostly migrated to Windows towards the end of the 1990s.

\subsection{Chronology of the Transitions}

The qualitative results gained from diskmags and newsgroups presented in the subsections above illustrate the mechanisms and characteristics of transitions. The weakness of such analysis is that we cannot perceive the magnitude and speed of migrations. To illustrate the chronology of events we plotted the data collected from the party results of 1992-2002 in time/productions coordinate system for visual inspection (Figure 1).

\section{Productions at major parties}

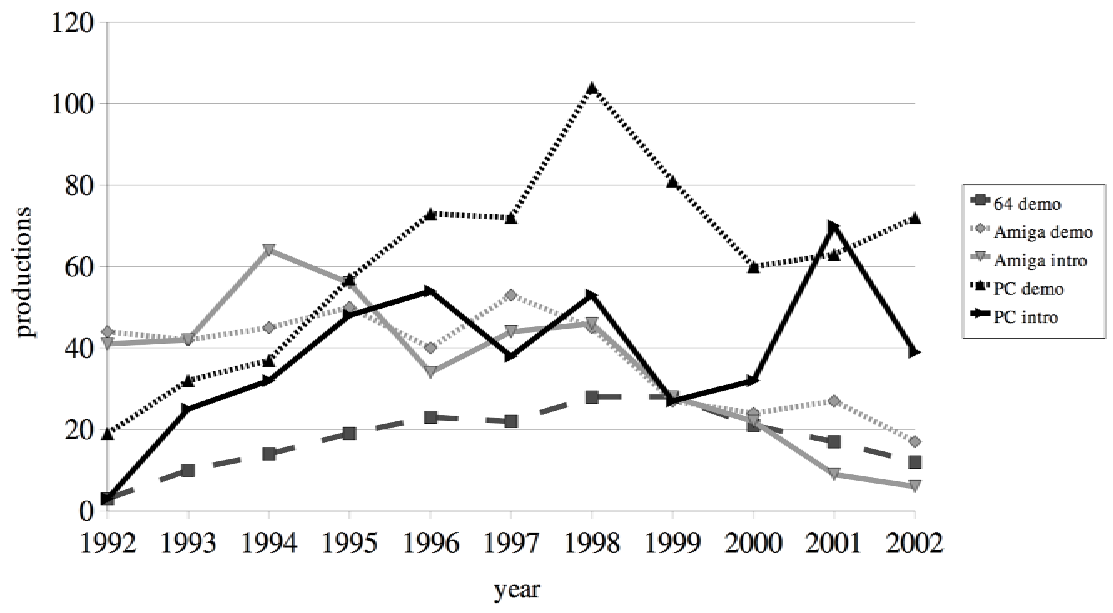

Fig. 1. Party productions by year 
Certain properties clearly stand out from the diagram and support the previous observations: until 1995, the PC scene was less productive than the Amiga scene; after 1996 the positions changed permanently. The drop after the peak year 1998 coincides with the Windows migration, presumably indicating the challenges involved in such transition. The decay of Amiga productions starts in 1998 and the trend continues during the following four years. Perhaps the most surprising observation is the Commodore 64 renaissance of the late 1990s. We can explain this phenomenon by the increase of Commodore 64 sceners attending the big mainstream parties, the decay of illegal activities, and the introduction of suitable competition categories in the parties. Additionally, such activity on an almost 20-year old machine reveals the strength of the retro/oldschool attitudes among the demoscene.

\section{Practices and Distinctions}

The demoscene reacts to large transitions through its own practices and its interpersonal relationships. The mechanisms both limit and enable the adaptation of new platforms. The same limitations and strengths apply when demoscene has to confront larger issues than the scene itself. Similarly, Rogers notes that the structure of a community can facilitate or impede the diffusion of innovations in a system [16].

\subsection{Skills}

As Turo-Kimmo Lehtonen [10] points out, the existing attachments between users and their technologies always both limit and enable new technologies. In the demoscene, an important form of attachment are the skills of a scener. Similar appreciation of skills is apparent in other male-dominated hobbyist communities as well $[21,11$, 5]. Technical features of a new platform do not automatically lead to success. Quite the contrary, too efficient a computer can undermine the skills needed to make demos. "I don't like lamers who require a hyper-fast PC to do little things like 24 faces glenz" (Imphobia \#7, 1993), a commentator notes of the PC. "I think using AGA just shows that you are incompetent to do really innovative things on normal machines" (R.A.W. \#6, 1993), another comments on the AGA technology on Amiga. However, this relation can also turn upside down: once the new computer starts to enable scene skills, it can be "a waste of talent" to stay with the old computer like the Amiga (Tsunami/VD in R.A.W. \#9, 1995).

The same applies to using new "too easy" tools, even with existing computers. The use of so-called demo-makers, tools that require little or no programming skills, was routinely frowned upon according to our data. Also certain interpreters like AMOS, a form of the BASIC programming language, were seen as not belonging in the scene: "We'll see a whole bunch of AMOS coders, but they hardly fit in our scene, do they?" (R.A.W. \#3, 1992). Nevertheless one could accept "easy" tools in the future, once they start to better enable scene skills. The role of the Flash scripting language for making demos is a topical debate on this.

Sceners often describe bad skills as something of a child's play. Since people frequently refer to demosceners as kids in the media [3], this distinction carries some irony. According to one diskmag, bad demos look like a drawing competition at a 
kindergarten (R.A.W. \#6, 1993). Another Amiga owner mentions that he would never buy a PC, "because this is indeed a computer for only playing, little children and for hardworking businessmen" (R.A.W. \#3, 1992). Therefore, the demoscene skills are not child's play, but they are not hard business either.

The most severe sign of no skills is ripping other demos' source code, graphics or music. The following newsgroup posting by Lancelot/Aggression in 1993 notes:

“ANYONE WHO COPIES PICTURES IS A LAMER!!! As a gfx-artist I am very well aware of how difficult it is to make a good picture. The reason why paintings are copied is that IT IS SO MUCH EASIER."

This frowning upon ripping is not a question of artist's copyright—with the demoscene's extensive cracking past, that would not be very believable. Rather, ripping is not a scene practice because it is too easy. The writer positions himself inside the scene: he is an artist and knows how difficult it is to make something that requires skills.

\subsection{Elites vs. Lamers}

The discussions we observed show that sceners put great efforts to define what they are not. This is marked by the distinction between "elites" and "lamers". Comparably, the early MIT hackers of the 1960s divided users to "winners" and "losers" [11]. The dictionary definition of "elite" points to the ambivalence of the concept. In common use, elite is a group of people considered best in particular society. However, in its original use in the late 18th century, elite was a noun of process: it meant "selection" or "choice". Similarly, in our data, many argue that it should go without saying what describes elite and lame. Nevertheless, the sceners still constantly debate this description. There seems indeed to be an active selection and choice of whom and what deserves to be elite, and even on what counts as a satisfactory test for that (see also Kline \& Pinch [8]).

The lamer has certain motivations, skills, and qualities. Firstly, the lamer is motivated by aims to be something that is not his essence. He would like to be famous or is trying to be better than he is. According to one account,

"The most typical lamer type is the guy in a group nobody knows because he has no contacts, moreover no coders, musicians, gfx... But this kind of lamers would like to be famous. But he isn't because he can't do anything." (Zine \#02, 1989)

The same account already points to lamer's skills, which we also covered in subsection 5.1. The lamer "can't do anything (related to the scene)". Other accounts mention that the lamers are constantly asking for advice on coding routines. Even more severe forms of lame skills are the buying or ripping of content made by other groups. Finally, lame qualities include having, aside little contacts, little ideas and personality: "it (lameness) is your personality or better the missing of it" (Imphobia \#3, 1992). With all these connotations, it was a powerful metaphor to call a new computer lame.

In our data, there is much more emphasis on defining lamers than on defining elites. There are many entire diskmag articles devoted to the motivations, skills and qualities of a lamer, none for the elites. This may reveal that the scene does not need 
to observe in detail the normal conditions. Rather, it needs to keep an eye on everything around the scene: all those changes that appear abnormal and even threatening to the scene's existence. For this purpose, the elites need the category of "the others", the lamers. As Domino/TRSI puts it in R.A.W. \#06 (1993), "What's the point in being elite if there are no lamers?"

\subsection{Confronting a Changing World}

We have underlined that demosceners have an active and self-reflective relation to new computers. They often consult other sceners and try to determine whether a new computer counts as viable for making demos. Similar patterns are apparent for example in studies by Lehtonen [10] and Rogers [16]. Most sceners are not enthusiastic early adopters of new technology, but want to use computers for certain ends instead. This means defining whether the computer in question is elite or lame and whether it enables scene skills to flourish. The sceners in our source material strived to create and maintain a critical distance to the new computers. The users were thus retaining a degree of control over technological change [10].

However, the degree of control over change was not high. The technological sphere still has an autonomy of its own. Similarly to the average consumers [10], the sceners were simply subjected to the arrival of Amigas and PCs-the scene could mediate the arrival, but not prevent it. Consequently, in our data, the sceners always eventually adopted the newest computers and technologies. In addition, while arguing about computers on more general level, the sceners often chose the same language as their environment: the language of the markets and companies. In a diskmag entry, "the death of Amiga" was said to result in "the stupid PCs and the videogames in charge of the computer and entertainment scene" (R.A.W. \#3, 1992). One commentator even claimed that Microsoft was "The innovative engine of the entire software industry. Does Microsoft support the Amiga? Microsoft supports any machine worth supporting” (R.A.W. \#8, 1995).

As we observed in subsection 5.1, the scene activities are sometimes contrasted to "hard business". However, the sceners can still use the arguments of markets for their own purposes. This inconsistent relationship with business describes the scene in relation to technological change. Internally, the scene can observe anything according to its own binary oppositions between elite and lame and skillful and non-skillful. Nevertheless, it cannot change the much larger spheres like the global markets of computers and the mass media. If the sceners want to discuss computer business, they can do nothing but communicate accordingly.

\section{Conclusion}

The transitions we have described illustrate the role of active communication in the domestication of home computers from the late 1980s to the late 1990s. People will reject a new platform at first if it does not fit the current community practices, no matter how technically advanced it is. One might expect that technically proficient and active people such as the demoscene members would be among the innovators or early adopters of new technology, but our study disproves such assumptions. The adaptation of community practices in the case of demoscene takes considerable time and largely follows the same pattern as any diffusion process. 
What appears in the mass media as harmless adolescents making audiovisual presentations hides an elaborate system of interpersonal relationships and practices. Thus portraying this complex community as a mere monoculture of nerds or a preschool for IT business [7] is an oversimplification. The conflicts both inside the scene and with the outside world may rather rudely affect the persons involved, because they are so deeply involved with the system. The emotional tone of the discussions also reveals the intimacy of computer as an object.

\section{References}

[1] Bagnall, B.: On the edge: the spectacular rise and fall of Commodore. Variant Press (2005)

[2] Google: Google Groups, comp.sys.ibm.pc.demos (2007), http: / / groups.google. com/group/comp. sys.ibm.pc.demos / about (accessed April 14, 2007)

[3] Green, D.: Demo or Die! Wired, issue 3.07 (1995)

[4] Gruetzmacher, T.: PC Demoscene FAQ, plain text version (v0.83) (2004), http://tomaes.32x.de/text/pcdemoscene_faq.txt (accessed April 14, 2007)

[5] Håpnes, T.: Not in Their Machines. How Hackers Transform Computers into Subcultural Artefacts. In: Lie, M., Sørensen, K.H. (eds.) Making Technology Our Own: Domesticating Technology into Everyday Life, pp. 121-150. Scandinavian University Press (1996)

[6] Inkinen, S., Salmi, M.: Media aseena ja työkaluna - hakkereita, teknohippejä ja koneromantiikkaa uuden median verkoissa (Media as a weapon and a tool - hackers, techno hippies and machine romanticism in the networks of new media). In: Tarkka, M., Hintikka, K.A., Mäkelä, A. (eds.) Johdatus uuteen mediaan (Introduction to new media), pp. 90-91. Edita (1996)

[7] Kauppinen, J.: Demoskenen alakulttuurista nousee suomalaisen it:n kärki (The leading Finnish IT springs from the demoscene subculture). Aamulehti 7.7.2005, p. 20 (2005)

[8] Kline, R., Pinch, T.: The social construction of technology. In: MacKenzie, D., Wajcman, J. (eds.) The Social Shaping of Technology, pp. 113-115. Open University Press (2004)

[9] Kurki, R.: WE ARE! Tutkimus postmodernista identiteetistä sukupuolittuneissa yhteisöissä (A study on the postmodern identity in gendered communities). Lahden ammattikorkeakoulu (Lahti Polytechnic) (2002)

[10] Lehtonen, T.-K.: The Domestication of New Technologies as a Set of Trials. Journal of Consumer Culture 3, 363-385 (2003)

[11] Levy, S.: Hackers. Dell Publishing (1994)

[12] Lönnblad, H.: Kahden tietokonedemon vertaileva analyysi (A comparative study of two computer demos). Musiikin Suunta 19(2), 28-34 (1997)

[13] Nordli, H.: The Net is not Enough: Searching for the Female Hacker. Norwegian University of Science and Technology (2003)

[14] Polgar, T.: Freax. The brief history of the demoscene, vol. 1. CSW Verlag (2005)

[15] Reunanen, M., Silvast, A.: The Demoscene Research Bibliography (2007), http: / / www. kameli.net/demoresearch (accessed October 25, 2007)

[16] Rogers, E.M.: Diffusion of Innovations, 4th edn. The Free Press, New York (1995)

[17] Saarikoski, P.: Valtavirtaa vastaan-Demoscene suomalaisen kotimikroilun historiassa (Against the mainstream-Demo scene in the history of the Finnish homecoputer hobbyists). Lähikuva 3/2001, pp. 54-65 (2001) 
[18] Saarikoski, P.: Koneen lumo. Mikrotietokoneharrastus Suomessa 1970-luvulta 1990luvun puoliväliin (The Lure of the Machine. The Personal Computer Interest in Finland from the 1970 s to the mid-1990s). Nykykulttuurin tutkimuskeskuksen julkaisuja 83 . Jyväskylä (2004)

[19] Shor, S., Eyal, A.: DEMOing: A new emerging art form or just another digital craft? (2002), http://rhizome.org/thread.rhiz?thread=8522 (accessed April $15,2007)$

[20] Tasajärvi, L. (ed.): Stamnes, B., Schustin, M.: Demoscene: the Art of Real-Time. Even Lake Studios \& katastro.fi (2004)

[21] Turkle, S.: The Second Self: Computers and the Human Spirit. Simon and Schuster (1984) 